\title{
Vous serez pris en photo
}

Pierre-André Doucet

De prime abord, se rendre compte lorsqu'on est de trop. Se situer dans l'espace. Cerner les angles, le langage.

«Minute, y’a tcheckun dans l'frame. »

Identifier le photographe, les objets. Peut-être un père, une famille. Clarifier sa position, sa relation avec eux. Avouer être l'intrus. Subir le malaise. Le rejet, l'humiliation. Chercher une porte de sortie. S'encabaner dans ses épaules. Tourner en rond. Faire la tortue. Fondre. Rougir.

«Un peu plus à gauche... Alright, guys, cheese! »

Paniquer. Tenter en vain d'éviter la lumière provenant de l'appareil. Entendre le déclic. Flash. Sentir la lueur vaporiser son aura, percuter sa peau, franchir la barrière cutanée et brûler le gras. S'amincir momentanément de honte. Faiblir sous le choc.

«Ah non, 'est pas bonne. On fait ça back.»

Comprendre que c'est encore de sa faute. Être hors cadre, l'accepter. Habiter sa marginalité.

Se rappeler qu'on a aussi eu une famille. Une mère. Un père, forcément, en quelque part. Des prétendants temporaires, de temps à autre. Essayer de reconstituer leur physique à partir de souvenirs refoulés. Permettre à son cerveau de générer un croquis sur papier jauni. Lutter contre son cœur qui se lève face à cette toxicité nouvellement déchaînée. Brièvement se tourner vers le Seigneur. Maudire ce processus, regretter ne pas avoir de représentations plus concrètes, plus saines à consulter.

Accepter que ses parents n'aient pas de photos de soi, à leur tour. Que ce n'est pas en raison de sa laideur ou de difformités innées. Qu'on a mérité d'être privé d'images. Qu'ils ont eu raison de négliger de commander des photos scolaires, de détruire les quelques albums de famille dans lesquels on figurait. Qu'on a été un fardeau insupportable, une tache qu'ils ont bien fait d'essuyer.

Se réconforter du fait qu'avant nous, des millions de personnes ont aussi vécu sans avoir été photographiées. Que même parmi les innombrables photos prises de nos jours, la vaste majorité ne sera jamais vue, appréciée. Que seules quelques-unes seront remarquées, conservées, chéries.

Passer des nuits blanches sur internet, à feuilleter des albums photo produits par d'autres. Lunes de miel, réunions de famille, événements rassembleurs. Actualités, mèmes, porno. Au fil du temps, malgré soi, sentir l'envie germer dans ses entrailles. Aspirer à cette immortalité particulière. Cliquer sur actualiser.

Un jour, suppurant de désir, repousser ses limites. Initier. Flâner devant une caméra. Rester dans les coins ombragés, par mesure de précaution. Veiller à atténuer les effets de la lumière. Adopter un air insouciant. Au moment crucial, détourner légèrement la tête, se frotter l'oreille. Flash. Constater que sa peau maintient sa couleur, que ses épaules ne s'élèvent pas. Percevoir le flash transformé momentanément en une lueur ardente, aguichante, mielleuse. Être saisi par cette nouvelle source d'énergie. Enfin vibrer d'un feu sacrificiel. 
Répéter l'exercice. S'immiscer dans d'autres cadres, remarquer des résultats similaires. S'immuniser progressivement aux brûlures du flash. À la longue, se réjouir. Poursuivre.

Au début, surprendre, faire rigoler. Secouer ses mains, cligner ses yeux. Sourire. Faire don de soi. Flash. Grandir. Observer silencieusement, impassible, tandis qu'on reprend le cliché.

S'introduire par subterfuge. Se camoufler. Se vêtir de verts, de bleus, de bruns. Faire la statue ou l'arbre entre deux têtes dans une photo de famille. Ne pas éveiller les soupçons. Se concentrer, se focaliser. Patienter tandis qu'on compose l'image, qu'on règle le focus, avant d'obstruer rapidement l'artefact ou le paysage. Viser le déclic. Flash. Étirer ses membres, prendre racine.

Peu à peu, réaliser à quel point on y prend plaisir. Jubiler lorsque son talon ou ses doigts briment la vue d'un tableau ou d'une muraille. Passer ainsi des après-midis entiers dans ses endroits préférés. Nourrir son feu. Lorsqu'en danger de s'en lasser, s'offrir de nouveaux lieux, de nouvelles contrées.

Cerner les endroits, les monuments, les occasions propices. Bazars, biennales, festivals. Carrousels, églises, ruines. Mariages, voyages, anniversaires. Consulter sites web et compiler listes. S'envoler au-delà d'un océan, s'il le faut. Se procurer un billet d'avion, réserver une chambre d'hôtel. Prendre congé et partir. Choisir des lieux étroits, bondés, aux proies faciles. L'Acropole. Tiananmen. Times Square. Sur les lieux, tendre son filet, traîner. Admirer son entourage. Récolter, se gaver. Pécher.

Exercer ses muscles maxillo-faciaux. Retrousser ses lèvres, gonfler ses joues, glacer ses yeux. Se pratiquer dans le miroir. Chez soi, dans le rétroviseur, chez le barbier. Être surpris lorsque ce dernier, un homme à la sexualité ambigüe, masse la base du crâne et les trapèzes à travers une serviette chaude, sans en avoir été demandé. Espérer. Croire à l'intimité. Y prendre goût, en jouir. Gémir. Se faire brusquement renvoyer.

Marcher, arpenter, péleriner. Peaufiner ses habiletés. Aiguiser son regard, se sensibiliser aux détails. Apprendre à dérégler les proportions, la luminosité, à déstabiliser des appareils par de légers coups. Apprivoiser le jeu des vélocités, le sens du timing. Traverser en avant-plan. Ruiner de front. S'assumer. S'imposer.

Voir naitre l'envie de conserver des souvenirs des corps et des visages que l'on croise. Passer de l'autre côté, prendre des gens en photo. Demander la permission, faire poli, rendre heureux. S'offusquer des refus occasionnels. Lécher ses plaies. Ses babines.

Se faire plaisir. S'emporter vers un lieu tropical aux palmiers bien entretenus. S'allonger sur une plage, s'abandonner au bercement des vagues, aux effleurements du vent. Se laisser pénétrer par les rayons du soleil sans se couvrir de lotion. Bronzer, plutôt que de rougir. Se baigner dans l'eau calme et chaude. Lorgner les bêtes qui défilent, consommer leur peau, leur joie. Convoiter. En accoster quelques unes, puis gérer les rebuffades, l'exclusion continue. Bouillir en solitude. Claquer la surface de l'eau de ses paumes. Pisser.

S'obstiner. Capturer les clichés que l'on veut. Prendre en otage des cuisses, des fourches. Des regards. Se frustrer lorsque les gens évitent le rayon de l'objectif, lorsqu'ils se dispersent comme un banc de poissons. Songer à la nécessité d'un complice ou d'un apprenti. Un dauphin. Évaluer les mécanismes nécessaires pour parvenir à l'embauche. Renoncer à l'idée.

Parfois, à la tombée de la nuit, traquer une victime dans un lieu isolé. Viser la palme. Chercher la lumière dans la noirceur. Suivre pour suivre.

Rebrousser chemin. Tout reconcevoir, recadrer. Revenir à la photobombe, mais en étant plus judicieux dans le choix de ses sujets. Des personnes courtes, de façon à favoriser l'occupation maximale de la photo en hauteur. Des femmes aux boucles volumineuses, aux hanches voluptueuses, aux seins libres. Des hommes aux allures de rugbymen, épaules et cou 
larges, oreilles protubérantes, coupe militaire. De préférence torses nus si le lieu et le climat le permettent. Pour varier, parfois de vieux couples aux traits androgynes ou des étudiantes asiatiques tournoyant leurs chevilles. Des enfants. Des gens qui occupent difficilement une pleine photo.

Se perdre avec eux dans les flashs. Perdurer.

En temps de pénurie des cibles de meilleure qualité, profiter de celles qui abondent. Les personnes saoules qui tentent d'éviter qu'on photographie leurs boissons. Flash. Les jeunes qui piaillent « selfie! ». Flash. Les touristes angoissés aux côtés des routards irrités. Flash, flash.

Se perfectionner davantage. Retoucher ses techniques, se munir de nouveaux moyens. Se déguiser. Se coiffer d'un chapeau, porter une moustache, des lunettes. Se vêtir d'un smoking d'agent secret, d'une robe à taille empire. De faux seins. De lentilles cornéennes rouges.

Refuser de sombrer dans l'oubli. Se servir. Rapprocher ses mains de la peau d'autrui, à leur insu. Sentir l'excitation des cellules proximales, des poils, des espaces vitaux qui s'entrechoquent. Vouloir, désirer un rapport. Lécher les cheveux d'une femme sans qu'elle s'en aperçoive. Tranquillement placer sa main sur le cul d'un homme. Souffler doucement sur une nuque, un lobe d'oreille. Chercher une bouche avec la sienne, des doigts avec les siens.

S'intensifier. Obliger l'attention. Gratter son nez, sa poche. Se déculotter. S'exhiber le cul, la queue. Flash. Multiplier les gestes vulgaires : un doigt d'honneur, une pipe simulée, une quenelle. Révolter.

Pénétrer les cadres par force. S'agiter. Échapper du café, tacher. Bousculer. Viser les points faibles: chevilles, creux de genoux, orteils. Cracher, gifler. Violenter.

Se radicaliser. Effectuer des recherches dans une chambre noire. Fixer un objectif. Dénicher du matériel, des outils. Modifier des appareils. S'armer.

Trouver un endroit branché, achalandé, médiatisé. Attendre le crépuscule. L’heure bleue. Anticiper la beauté des contrastes sur les négatifs à venir. Resserrer ses lèvres en un demi-sourire.

Placer le dispositif. Créer une diversion. Hurler, gesticuler, empoigner son pectoral gauche. Regrouper. Attrouper. Appuyer sur le déclic. Boom. Sinistrer, ruiner.

Accueillir chaleureusement les flashs qui inondent l'espace. S'y noyer. S'immoler. S'immortaliser. Planer vers la une par ce carnage lumineux. Réussir.

Vous écrirez alors dans la lumière.

Vous serez pris en photo. 